\title{
Reducing the manual length setting error of a passive Gough-Stewart platform for surgical template fabrication using a digital
} measurement system

\begin{abstract}
As recently demonstrated, a passive Gough-Stewart platform (a.k.a. hexapod) can be used to create a personalized surgical template to achieve minimally invasive access to the cochlea. The legs of the hexapod are manually adjusted to the desired length, which must be read off an analog scale. Previous experiments have shown that manual length setting of the hexapod's legs is error-prone because of the imprecise readability of the analog scale.

The objective of this study is to determine if integration of a linear encoder and digitally displaying the measured length help reduce the length setting error. Two experiments were conducted where users set the leg length manually. In both experiments, the users were asked to set the leg length to 20 nominal values using the whole setting range from $0 \mathrm{~mm}$ to $10 \mathrm{~mm}$. In the first experiment, users had to rely only on the analog scale; in the second experiment, the electronic display additionally showed the user the actual leg length.

Results show that the mean length setting error without using the digital display and only relying on the analog scale was $(0.036 \pm 0.020) \mathrm{mm}$ (max: $0.107 \mathrm{~mm})$ in contrast to $(0.001$ $\pm 0.000) \mathrm{mm}$ (max: $0.002 \mathrm{~mm}$ ) for the experiment with the integrated digital measurement system. The results support integration of digital length measurement systems as a promising tool to increase the accuracy of surgical template fabrication and thereby patients' safety. Future studies must be
\end{abstract}

\footnotetext{
*Corresponding author: Julia Kilian: Department of Otolaryngology and Cluster of Excellence EXC 2177/1 “Hearing4all", Hannover Medical School, Stadtfelddamm 34, Hanover, Germany, kilian.julia@mh-hannover.de Max-Heinrich Laves, Tobias Ortmaier: Institute of Mechatronic Systems, Leibniz University Hannover, Hanover, Germany Tobias Blum, Thomas Lenarz, Thomas S. Rau: Department of Otolaryngology and Cluster of Excellence EXC 2177/1 "Hearing4all", Hannover Medical School, Hanover, Germany
}

conducted to evaluate if integration of a linear encoder in each of the six legs is feasible.

Keywords: hexapod, accuracy setting, length setting error, linear encoder, digital display, length measurement

https://doi.org/10.1515/cdbme-2021-2023

\section{Introduction}

Microstereotactic frames (MSF) are under development to enable highly precise drilling of a surgical access based on a preoperative, individually planned trajectory. This concept has been especially investigated and described in the context of minimally invasive cochlear implant surgery or neurosurgical interventions. A recently proposed type of a MSF consists of a uniform, reusable reference frame and a personalized surgical template $[1,2]$ (see Figure 1). The reference frame is bone-anchored to the patient's head and the surgical template is individually assembled from few separate parts and bone cement, which permanently fixes the instrument guide in the planned orientation. To achieve the right orientation of the multi-piece template, an alignment tool is necessary. In its current design, such alignment tool is based on a GoughStewart platform (hexapod) with manually adjustable leg lengths (see Figure 2). The template's baseplate is mounted on top of the moving platform of the hexapod. The top platform includes two dowel pins and a threaded hole, which is an identical mounting interface as on top of the reference frame. This allows an exact fit and fixation of the surgical template both on the reference frame and the alignment tool. The individual trajectory is represented by a vertical centering pin which is installed inside the hexapod. To set the desired pose of the top platform, six leg lengths are planned and need to be manually adjusted. To set the length of the legs each leg 
Reducing the manual length setting error of a passive Gough-Stewart platform for surgical template fabrication using a digital measurement system

features a micrometer screw with an analog nonius scale. This step is herein after referred to simply as "length setting".

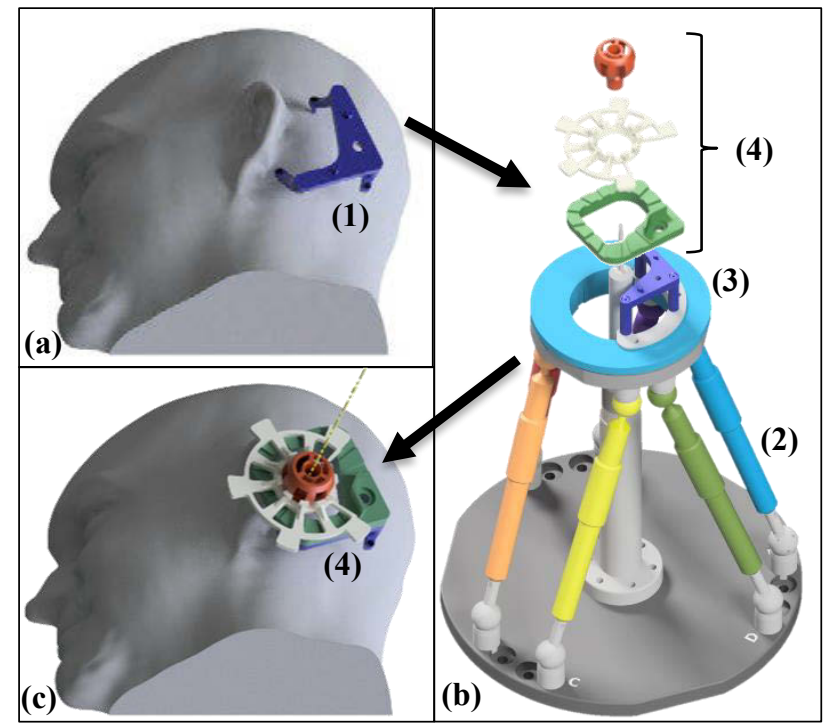

Figure 1: (a) Bone-anchored reference frame (1), (b) alignment device (2) with mounting interface (3) for the surgical template (4), (c) Surgical template mounted to the reference frame

Previous experiments have shown that manual length setting is a crucial source of individual errors [1]. The value set on the analog scale is subjective and differs depending on the point of view, the user's carefulness and familiarity with that type of analog scale.

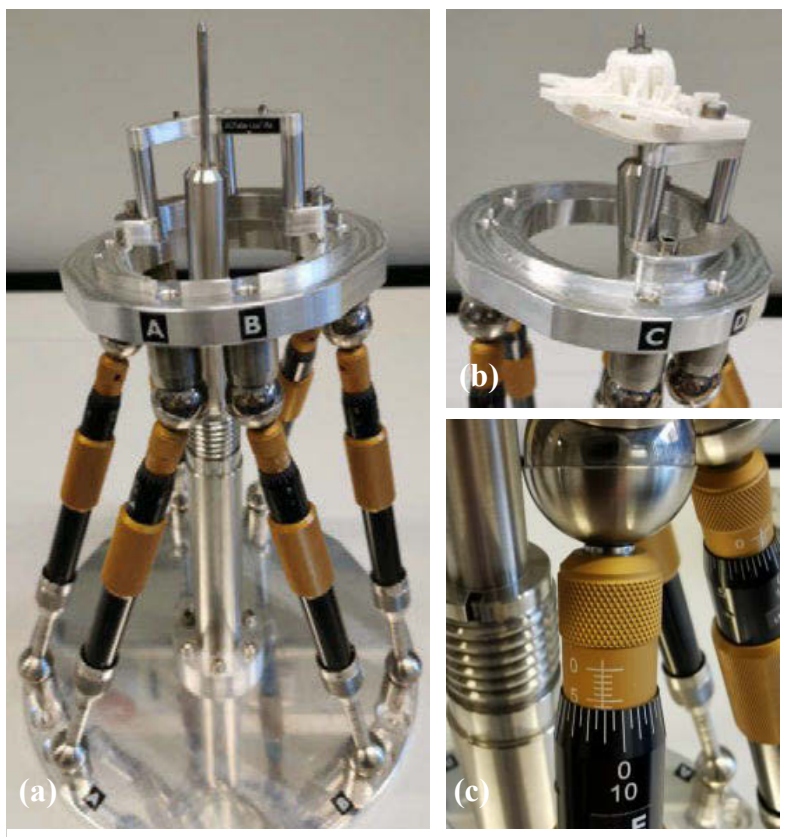

Figure 2: (a) Hexapod with six manually adjustable legs and a central alignment pin. (b) The surgical template is mounted on top of the moving platform of the hexapod. The pose of the platform is set by adjusting the leg length. (c) The analog scale on each leg is used to set the planned value by turning the micrometer screw in a range between $0 \mathrm{~mm}$ and $10 \mathrm{~mm}$
The aim of this study is to determine if the integration of a digital measurement system will (a) help users to achieve a better average accuracy in leg length setting and (b) can prevent severe maladjustments (as e.g., caused by transposed digits) compared to relying only on the analog scale. We also evaluate how many decimal places can be set accurately by using the micrometer screw to set the leg length. A length setting accuracy of $0.05 \mathrm{~mm}$ for each leg is required to achieve a highly precise orientation of the surgical template.

\section{Material \& Methods}

\subsection{Experimental Setup}

To evaluate whether a digital measurement system is a promising possibility to increase the length setting accuracy, the following experimental setup was constructed. The leg of a passive hexapod (X1med3D, SchickDental GmbH, Schemmerhofen, Germany) was dismounted and fixated on a 3D-printed setup. This allows more space to integrate the length measurement system compared to adapting the system to an installed leg of the hexapod. An absolute linear encoder was used to measure the length change of the hexapods legs (Heidenhain LC 415, accuracy: $\pm 0.005 \mathrm{~mm}$ ). The system is based on optical scanning of absolute graduations. The linear encoder was mounted parallel to one of the hexapod's legs, as shown in Figure 3, to measure the translational length change while turning the micrometer screw. The length measurement system was connected to a digital display (Heidenhain GAGECHEK 2013) to visualize the absolute value of the leg length in millimeters with four decimal places.

\subsection{Experimental evaluation}

A user study with two experiment types and six users was conducted. In both experiments, the users were asked to set the leg length to 20 nominal values spread over the whole setting range. The users were seated in front of the experimental setup. Five of the users did not have any previous experience in manual leg length setting of the hexapod legs. The length setting was done by turning the micrometer screws and the variable length of the hexapod leg can vary between $0 \mathrm{~mm}$ and $10 \mathrm{~mm}$. In the first experiment, users had to set 20 randomly defined values between $0 \mathrm{~mm}$ and $10 \mathrm{~mm}$ by only relying on the analog scale. The length measurement system and digital display are also connected to the leg, but the display was not visible for the users in this first experiment. The set leg lengths 
Reducing the manual length setting error of a passive Gough-Stewart platform for surgical template fabrication using a digital measurement system

were documented by an independent investigator. The digital values were used as the reference value and considered as the true leg length. In a second experiment the users had to set the same 20 nominal values but this time they had to rely only on the digital display.

They were asked to adjust as well as possible all four visible decimal places to the predefined values by carefully turning the leg back and forth. There was no time limit for that task, but the users were asked to notify when they felt that no further improvement was possible.
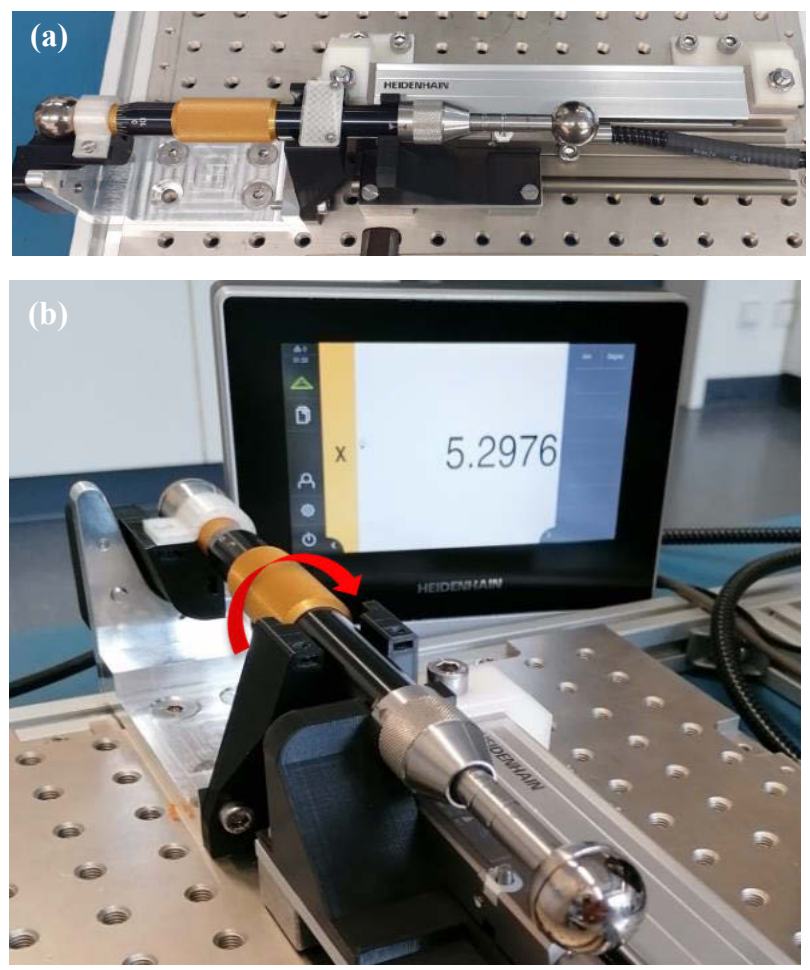

Figure 3: (a) The optical absolute length measurement system which is connected to one of the hexapod legs. (b) The experimental setup: The digital display is connected to the measurement system and shows the adjusted leg length. The leg is twisted at the middle part in the shown direction to change the leg length

\section{Results}

For each experiment a total of 120 nominal values ( 20 values times six users) were used for the evaluation. The mean length setting error in the first experiment was $(0.036 \pm 0.020) \mathrm{mm}$ whereas for the second experiment it was $(0.001 \pm 0.000) \mathrm{mm}$. In Figure 4 the deviation from the nominal values of the two experiments are compared and plotted in a boxplot on a logarithmic scale due to the large differences in the mean values. The experiment with "analog" length setting revealed in 24 of 120 cases $(20 \%)$ a threshold of $0.05 \mathrm{~mm}$ for the length setting accuracy was exceeded. The maximum deviation from the nominal value in that experiment was found to be 0.107 mm. In contrast, when using the "digitized" method the accuracy limit was never exceeded, with a maximum length setting error of $0.002 \mathrm{~mm}$.

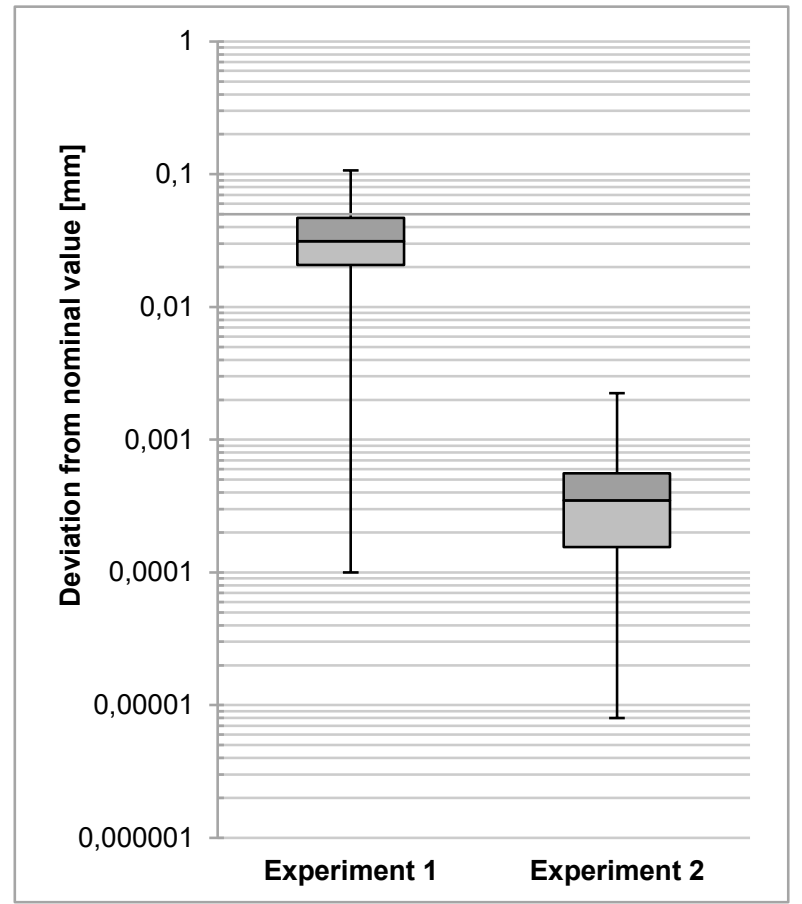

Figure 4: Boxplot of the length setting error in the two conducted experiments: In experiment 1 the users had to rely only on the analog scale on the hexapods leg whereas in experiment 2 the users could see the actual length on an electronic display

\section{Discussion}

The proposed microstereotactic frame comprises an individually fabricated surgical template to be produced directly in the OR. Provided freehanded assembly of its components (based on the previously planned trajectory) cannot be done accurately, an alignment device for temporary pose setting is required until all parts are irreversibly glued together. To keep the overall system simple and inexpensive, a manually operated alignment device is proposed. Pose setting is achieved by adjusting the length of all six legs of the hexapod (see Figure 2). As the accuracy of pose setting directly impacts the drilling accuracy when using the surgical template, accurate length setting is essential for the safety of the patient.

In order to address previously reported errors [1] in manual operation of the pure mechanical hexapod's legs (micrometer 
Reducing the manual length setting error of a passive Gough-Stewart platform for surgical template fabrication using a digital measurement system

screws) we aimed to integrate a digital length measurement system as one possibility to exclude operating errors and to achieve the required reliability and safety. As expected, the average length setting error was significantly smaller when the users took advantage of the digitally displayed actual leg length compared with the conventional reading of the analog scale on the micrometer screw of the hexapod leg. In other words, the problem of poor readability of the analog scale can be effectively overcome by the method incorporating the digital display. By utilizing the digital length measurement system the maximum length setting error was less than $0.002 \mathrm{~mm}$, which is far below the requirement of $0.05 \mathrm{~mm}-$ hence more accurate.

Alternatively, one could try other designs for the length setting mechanism with better readable scales. This would keep the costs comparably low. However, reading of an analog scale, even an improved one, will be always more difficult, subjective and time consuming than reading a digital display. This is especially true when the adjusted values of all six legs need to be compared with the planning. Furthermore, controlling by a second person (four-eyes principle) is easier and more valid if the values are presented as digital numbers. Finally, the digital availability of the measured values allows further processing, such as providing an optical or acoustical feedback when the whole hexapod is correctly adjusted as a prerequisite to further continue the template fabrication.

One could argue that a fully automated hexapod for template fabrication could also guarantee highly accurate pose setting and provide feedback to the user regarding the readiness for template fabrication. In such a case, both the motion and the position measurement could be replaced by technical components, such as an actuator and a sensor, respectively. However, changing the length of a linear or helical joint can be done manually in a sufficiently accurate manner. In contrast, estimation of the accurate length is beyond human capability. Therefore, with respect to overall costs we consider complete automation of the alignment device only as a last resort if our approach fails in further evaluation experiments.

Based on the presented results, we conclude that integration of length measurement systems in the alignment device seems to be a promising tool to decrease the length setting error and thereby increase the accuracy of the surgical template fabrication. Furthermore, our experience with the digital display and the users' feedback indicate that it is an impactful method to obviate serious individual length setting errors, as observed in [1].

However, the experimental setup needed a lot of space and the integration of the linear encoder in one of the hexapod legs was only possible with a dismounted leg and additional setup. To integrate a length measurement system in each of the hexapod legs, a smaller system which fits into the limited assembly space between the six legs of the hexapod is necessary. It also must be evaluated if the additional costs for incorporating a digital measurement system and the required effort exceed the benefits of increased accuracy for the particular application. It may be a good tradeoff between costs and accuracy in comparison to a fully automated hexapod used for template fabrication. Future studies have to be conducted to determine if the integration of a linear encoder in each of the six hexapod legs is feasible with regard to assembly space, costs and applicability in the operating room.

\section{Author Statement}

Research funding: This work was funded by the German Research Foundation (DFG, RA 2751/4-1 and under Germany's Excellence Strategy - EXC 2177/1 - Project ID 390895286).

We thank Maria Geraldine Zuniga Manrique for the provided constructive criticism for this work.

Ethical approval: The conducted research is not related to either human or animal use.

Conflict of interest: Authors state no conflict of interest.

\section{References}

[1] Rau Th. S., Lexow, G. J., Blume D., Kluge M., Lenarz Th., Majdani O. Micro-stereotactic frame utilizing bone cement for individual fabrication. An initial investigation of its accuracy. Proc. SPIE 10135, Medical Imaging 2017: Image-Guided Procedures, Robotic Interventions, and Modeling, 10135.

[2] Rau Th. S., Witte S., Uhlenbusch L., Kahrs L.A., Lenarz Th., Majdani O. (2021): Concept description and accuracy evaluation of a moldable surgical targeting system. J. Med. Imag. 8(1), 015003. 